\title{
High-Energy Neutrino Emission from Blazars
}

\author{
Foteini Oikonomou ${ }^{a *}$ \\ ${ }^{a}$ Institutt for Fysikk, Norwegian University of Science and Technology, Trondheim, Norway \\ E-mail: foteini.oikonomou@ntnu.no
}

Active galactic nuclei (AGN) with relativistic jets are the most powerful persistent astrophysical sources of electromagnetic radiation in the Universe. Blazars are the most extreme subclass of AGN with jets directed along the line of sight of the observer. Their high-energy photon emission dominates the extragalactic gamma-ray sky and reaches multi-TeV energies. This demonstrates that blazars accelerate particles to very high energies. It has long been suspected that blazars may also accelerate protons to very high energies and thus be cosmic neutrino sources. Being extremely rare objects in addition to being bright, blazars are among the most readily testable neutrino candidate source classes. Several multi-messenger monitoring campaigns have recently been triggered in response to high-energy neutrinos observed with the IceCube Neutrino Observatory from the direction of blazars. In this contribution, I summarise the theoretical interpretation of these observations and give an overview of the possible role of blazars as neutrino sources in light of the experimental results.

$37^{\text {th }}$ International Cosmic Ray Conference (ICRC 2021)

July 12 th - 23rd, 2021

Online - Berlin, Germany

\footnotetext{
${ }^{*}$ Presenter
} 


\section{Introduction}

The discovery of high-energy astrophysical neutrinos was reported in 2012 by the IceCube Neutrino Observatory [1]. Since then, the field of neutrino astronomy has made considerable progress, with the detection of neutrinos in multiple analysis channels [2,3], and with the first indication of a joint source of $\gamma$-rays and neutrinos [4]. In tandem with these experimental breakthroughs, significant theoretical efforts are being made to determine the origin of astrophysical neutrinos. The proposed neutrino source classes include Galactic sources, $\gamma$-ray bursts, jetted and non-jetted Active Galactic Nuclei as well as calorimetric environments such as starburst galaxies to name some of the possibilities [5-9]. The isotropy of the neutrino arrival direction distribution rules out a purely Galactic origin and implies that at least $86 \%$ of the neutrinos have an extragalactic origin.

A particularly promising source candidate are blazars. These are AGN with jets in our line of sight. Blazars are very rare objects. Only a small fraction of galaxies host an AGN with a relativistic jet, and only $\sim 1 / 100$ of them point in our line of sight. Thus, the number density of blazars inferred with the Fermi Large Area Telescope is approximately $\lesssim 10^{-6} \mathrm{Mpc}^{-3}$ [10]. The apparent luminosity of a powerful blazar can be of order $10^{49} \mathrm{erg} / \mathrm{s}, 10^{7}$ times larger than the luminosity of the Milky Way. Here the luminosity is the apparent luminosity, which benefits from a relativistic boost in our direction. A similar boost is experienced for neutrinos if they are produced in blazar jets.

Blazars are the dominant sources of $\mathrm{GeV}$ and $\mathrm{TeV} \gamma$-rays. This means that as a population they are excellent high-energy accelerators and that they comfortably accelerate particles to above $100 \mathrm{TeV}$. It is reasonable to assume that some of the accelerated particles are protons, in which case, if interactions with ambient matter or photons occur, high-energy neutrinos will be produced. Thus, blazars have been proposed since the early nineties as ideal sources of neutrinos [11-26].

This proceeding summarises the key points of the highlight talk I gave at the ICRC 2021. In Section 2, a brief overview of neutrino production mechanisms in blazars is given. In Section 3 the current state of knowledge of the possible contribution of blazars to the diffuse neutrino flux measured with IceCube is reviewed. In Section 4, the capabilities of blazars to be detected as neutrino point sources is reviewed, with a focus on three recently observed neutrino-blazar associations for which detailed modelling was performed. A discussion and outlook follow in Section 5 .

\section{Neutrino production in blazars}

Neutrinos are produced in photopion $\left(p \gamma, p+\gamma \rightarrow \pi^{+}+n\right)$ or hadronic $\left(p p, p+p \rightarrow X+N_{\pi} \pi^{ \pm}\right)$ interactions of protons and nuclei. Here $N_{\pi}$ is the pion multiplicity. Jetted AGN possess strong radiation fields, particularly at distances close to the base of the jet, associated with emission from the accretion disk surrounding the supermassive black hole (SMBH), but also within the jet itself. Hence, photopion interactions are likely if protons are accelerated by blazars. Some possibilities exist for $p p$ interactions, for example, if dust clouds or a star intersect with accelerated protons, but in general, the $p \gamma$ process is thought to be the prevalent process. An illustration of this, often assumed, scenario is shown in Fig. 1. Here, a spherical emitting region is comoving with the jet which has bulk Lorentz factor $\Gamma_{\text {jet }}$ which is typically inferred to be in the range $\sim 10-50$. 
Usually, four target photon fields are considered; jet radiation, the radiation from the accretion disk, intercepted accretion-disk radiation reradiated in the ultraviolet range by fast-moving gas clouds (known as the broad-line region), and, further away from the jet base, intercepted accretion-disk radiation reradiated by the dust torus at infrared wavelengths.

Of the energy lost by protons with energy $\varepsilon_{p}$ in $p \gamma$ interactions, about 3/8ths go to neutrinos, resulting in the production of neutrinos with all-flavour luminosity, $\varepsilon_{v} L_{\varepsilon_{v}}=3 / 8 f_{p \gamma} \varepsilon_{p} L_{\varepsilon_{p}}$. Here, $f_{p \gamma}$ is the photopion production efficiency, which depends on the number density of available photon targets and the distance over which the interactions take place and $\varepsilon_{v / p} L_{\varepsilon_{\nu / p}}$ is the luminosity per logarithmic energy of neutrinos and protons respectively. Each neutrino is produced with energy $\varepsilon_{v} \sim 0.05 \varepsilon_{p}$. The remaining 5/8ths of energy lost by protons result in the production of electrons and $\gamma$-rays, which generally leads to an electromagnetic cascade inside the accelerator which reemerges at keV-GeV wavelengths [27]. Thus, neutrino production is accompanied by X-ray and $\gamma$-ray emission with comparable luminosity.

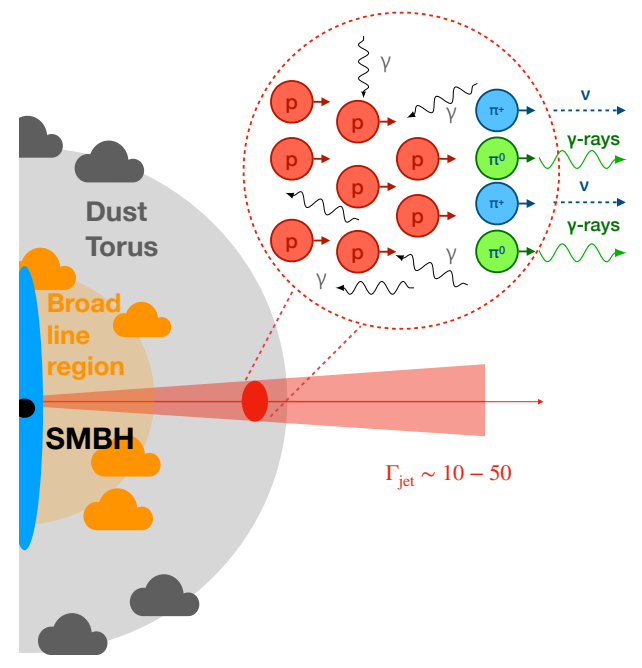

Figure 1: Schematic representation of neutrino production in a blazar jet through $p \gamma$ interactions. High-energy protons present in the emitting region interact with photons to produce charged and neutral pions leading to comparable fluxes of neutrinos and $\gamma$-rays.
The proton luminosity, $L_{p}=\int_{\varepsilon_{p}} L_{\varepsilon_{p}} d \varepsilon_{p}$, is a key unknown quantity for neutrino model predictions. Upper bounds on it can be derived from electromagnetic (EM), neutrino, and ultra-high energy cosmic ray (UHECR) observations and will be discussed below. The exact value of $f_{p \gamma}$ is also unknown but bounds on this quantity are tighter since in general, it can be determined from the shape of the $\gamma$-ray spectrum whether $\gamma$-rays escape the blazar without suffering severe internal attenuation on the same photon fields that facilitate $p \gamma$ interactions [27-29].

If blazars facilitate intense neutrino production, the EM cascade radiation is an additional spectral signature [30]. Alternatively, the absence of EM cascade radiation can be used to set limits on the proton luminosity and thus on the neutrino output of the sources. As was demonstrated in the case of TXS 0506+056, the absence of EM cascade radiation provides a stronger constraint on the high-energy proton content of the blazar jet than the detection of a single high-energy neutrino [31-34].

Fig. 2 gives a schematic summary of the neutrino spectrum produced in photopion interactions of a proton population with a distribution that follows $\mathrm{d} N / \mathrm{d} \varepsilon_{p} \propto \varepsilon_{p}^{-2}$, with photon fields typical in a blazar. The result is a highly peaked neutrino spectrum in terms of energy flux, even though the proton spectrum is flat. This is due to the resonant shape of the $p \gamma$ cross-section and the decreasing number of photons as a function of energy which is typical in blazars.

If protons of sufficiently high-energy are present in the jet of a blazar at redshift $z$, the typical energy of the emerging neutrinos is $\varepsilon_{v} \approx 1 \mathrm{PeV}\left(40 \mathrm{eV} / \varepsilon_{t}\right)(1+z)^{2}$, for interactions with photons with energy $\varepsilon_{t}$ which are stationary with respect to the SMBH. Quoted energies are in the observer's frame. For target photons comoving with a jet with bulk Lorentz factor $\Gamma$, the emerging neutrino energy is $\varepsilon_{v} \approx 100 \mathrm{PeV}\left(40 \mathrm{eV} / \varepsilon_{t}\right)(\Gamma / 10)^{2}(1+z)^{2}$. 

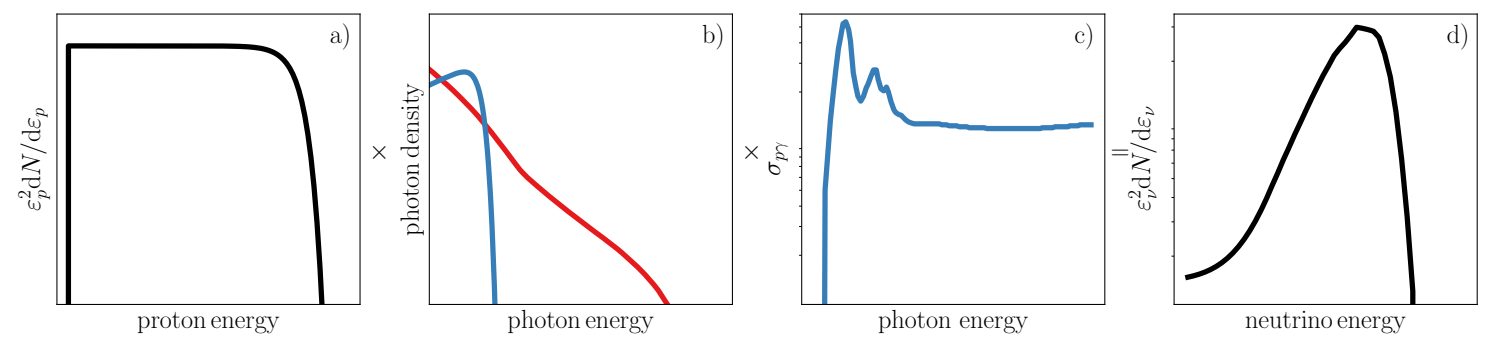

Figure 2: Schematic overview of the expected neutrino spectrum of blazars from $p \gamma$ interactions. A population of protons with a spectrum that evolves as $\mathrm{d} N / \mathrm{d} \varepsilon_{p}=\varepsilon^{-2} \exp \left(-\varepsilon_{p} / \varepsilon_{p \text {,max }}\right)$ (panel a) interacts with the photons from the blazar jet (red) and possibly stationary photons with a thermal spectrum (blue) in addition (panel b). Such thermal photon fields exist close to the base of many AGN jets and are reprocessed radiation from the accretion disk. Due to the resonant shape of the $p \gamma$ cross-section (panel c) and the decreasing number of photons as a function of energy, higher energy protons have access to a larger number of photon targets giving rise to a strongly peaked neutrino spectrum (panel d). All the axes are in doublelogarithmic scale.

\section{Blazars as sources of the diffuse IceCube neutrino flux}

Figure 3 shows the measured neutrino flux and the predicted diffuse flux of high-energy neutrinos in different blazar models. Although the models were developed during a period of almost 20 years and are based on different assumptions, the overall shape and peak energy of the expected neutrino flux among them are comparable. All the models predict that the blazar neutrino flux peaks at $>10 \mathrm{PeV}$ for the reasons explained above concerning the neutrino production mechanism. This is well beyond the energy range in which the bulk of IceCube neutrinos have been observed, and the observed spectral index of the diffuse astrophysical neutrino flux is significantly softer than the blazar models predict. At the time of writing, the best-fit spectral index is $E^{-2.37 \pm 0.09}$ for the through-going muon neutrino flux, and even softer for the high-energy-starting-event and cascade samples [2, 3, 39].

The normalisation of the neutrino flux in the majority of the models is a semi-free parameter, and it is proportional to $L_{p}$. It is constrained by the non-observation of $>10 \mathrm{PeV}$ neutrinos with IceCube and the Pierre Auger Observatory (Auger). The baryon loading factor, defined as $\xi=L_{p} / L_{\gamma}$, where $L_{\gamma}$ is the $\gamma$-ray luminosity of the blazar, is often used to characterise the proton content of the jet. The absence of $>10 \mathrm{PeV}$ neutrinos thus far, in combination with the model of [18], implies that the proton content of blazar jets on average must be $\xi<50$. Different model assumptions lead to slightly different limits on $\xi$.

The upper limits on the all-blazar neutrino flux obtained with IceCube and Auger also constrain the origin of $\gamma$-rays in the blazar population. For example, in the model of [22] the neutrino flux is assumed to be proportional to the $\gamma$-ray flux and it is parametrised by the quantity $F_{\gamma} / F_{\gamma}$ which is the ratio of the neutrino to $\gamma$-ray flux. The current upper limit of the $F_{v} / F_{\gamma}$ ratio of 0.08 [3], implies that at most a few percent of all detected blazar $\gamma$-ray emission is produced in photohadronic interactions.

Figure 3 also shows the $90 \%$ CL upper limit on the contribution of $\gamma$-ray selected (from the 3FHL catalogue [40]) blazars to the IceCube neutrino flux for an assumed $E^{-2.19}$ power-law 


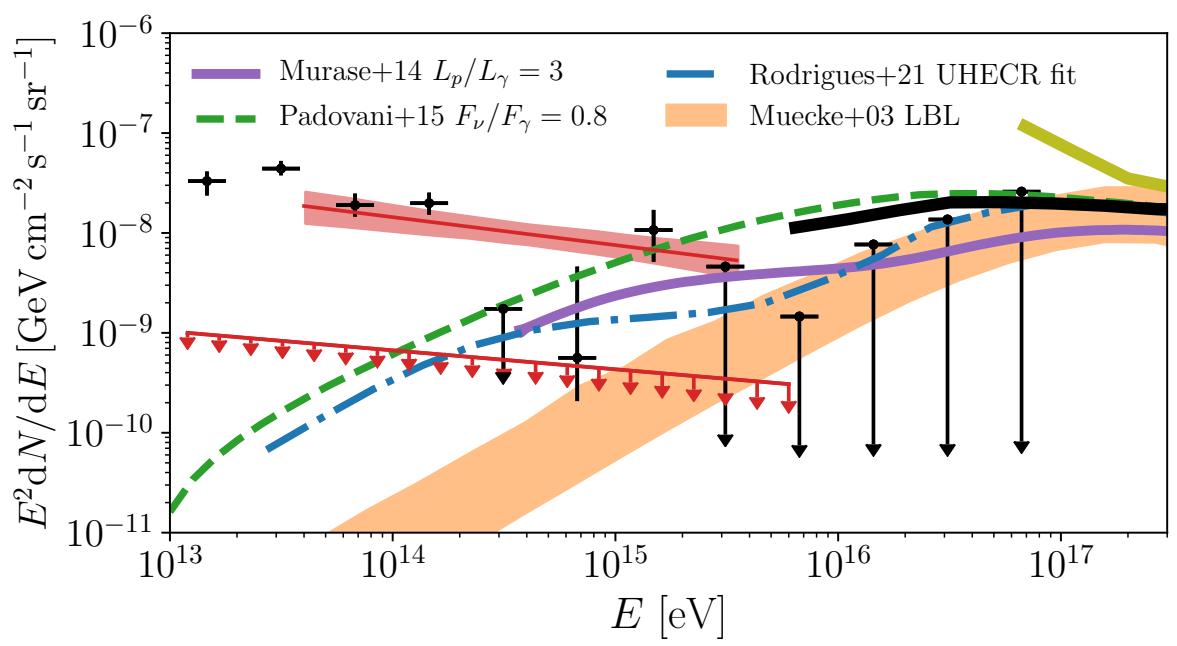

Figure 3: Measured neutrino flux, and predicted diffuse flux of high-energy neutrinos in different blazar models. The red area corresponds to the best-fit and $1 \sigma$ uncertainty region of the through-going muon neutrino flux measured with IceCube from [35]. The black data points correspond to the high-energystarting-event energy spectrum measured with IceCube [2]. The black solid line gives the 90\% CL upper limit obtained with nine years of data with the IceCube extremely-high-energy analysis [36]. The yellow line gives the $90 \%$ CL upper limit on the diffuse neutrino flux from the Pierre Auger Observatory [37]. The red solid line shows the $90 \%$ CL upper limit on the average neutrino flux of all blazars from the stacking analysis of [38]. The blazar neutrino models shown correspond to the expected diffuse flux from [14] (orange band), all-blazar model normalised to the diffuse UHECR flux (corresponding to baryon loading factor $\xi=3$ ) [18] (purple solid line), all-blazar model of [22] (green dashed line), and all-blazar model from a fit to the UHECR spectrum and composition observables of [26] (blue dot-dashed line).

spectrum, based on the stacking analysis of [38]. The limit depends on the assumed spectral index of the neutrinos but constrains the relative contribution of the blazars to $<16.7 \%$ of the total IceCube flux. As will be summarised in Section 5, stacking analyses, through which it is investigated whether there is an excess of neutrinos from the directions of the ensemble of resolved sources from a particular population, are powerful and have already strongly constrained several promising candidate source classes as the dominant sources of high-energy astrophysical neutrinos in addition to blazars. Independent upper limits to the relative contribution of blazars to the diffuse neutrino flux have been derived from the absence of clustering and of neutrino point sources in the IceCube data [41-44], and also disfavour blazars as dominant sources of the IceCube neutrinos.

The limits on blazars as dominant sources of the IceCube neutrino flux are severe but can be avoided if these blazars which are unresolved in $\gamma$-rays provide overwhelmingly more neutrinos than the brighter, observed ones, with factors of $\xi=10^{7}$ required for the faintest blazars during their longterm emission [25]. However, for well-studied blazars, such values of $\xi$ are ruled out based on the absence of a corresponding spectral signature in X-ray and $\gamma$-rays. In addition, such a model demands that $\mathrm{CR}$ acceleration stops at low energies. As a result, blazars cannot contribute to the diffuse UHECRs in a scenario that avoids the IceCube stacking limits.

It has been pointed out that the observed high-energy neutrinos, UHECRs and $\gamma$-rays indicate comparable luminosity densities. This apparent coincidence has been discussed as a possible 


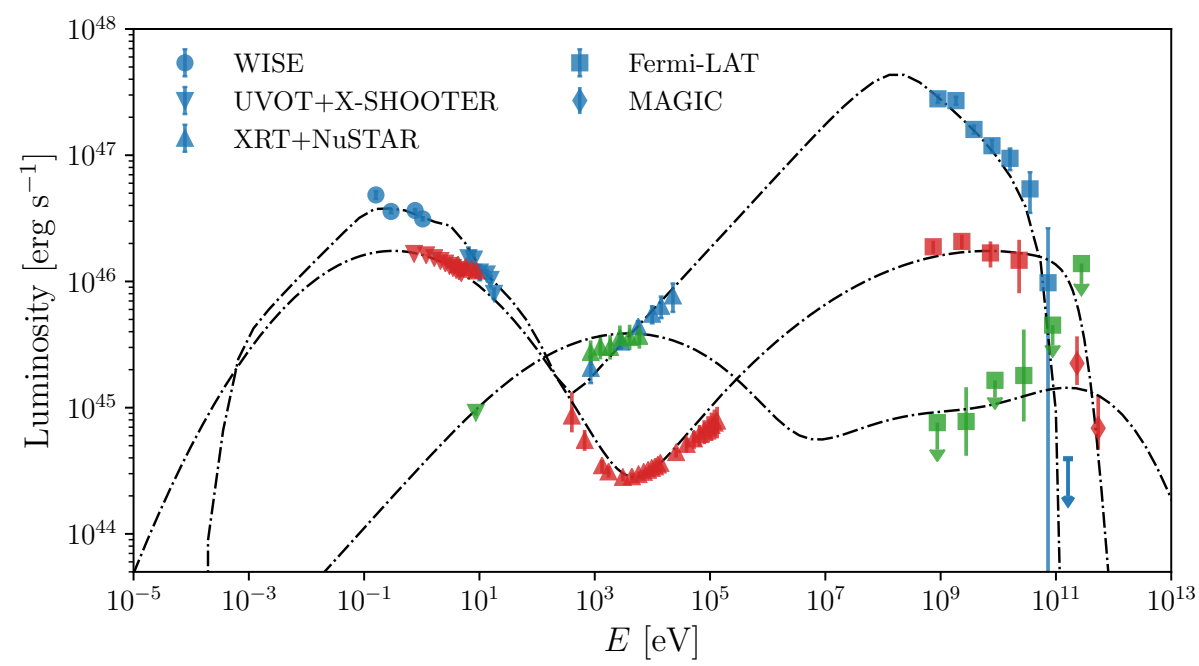

Figure 4: The spectral energy distributions of the blazars TXS 0506+056 (red), PKS 1502+106 (blue), and 3HSP J095507.9+355101 (green) at the time of arrival of IC-190730A, IC-170922A, and IC-200107A respectively, plotted in terms of the intrinsic source luminosity. The model fits are from Refs. [51-53]. Detailed references to the observations can be found therein.

indication of a joint origin of the three messengers [45-47]. However, the majority of $\gamma$-rays originate in blazars [48-50] and analyses of IceCube data all but exclude a dominant contribution of blazars to the neutrino flux below $100 \mathrm{TeV}$. Therefore, a common origin of the bulk of all the three messengers seems unlikely.

\section{Blazars as powerful neutrino point-sources}

Even though the majority of experimental results suggest that blazars are not the dominant sources of the neutrinos that IceCube has measured thus far, being very rare and extremely bright, blazars may still be detectable neutrino point sources. Some indications of correlations of blazars with high-energy neutrinos have been reported in [57-59], (see also [60]). If corroborated by future observations, these results would suggest that a fraction of the neutrino flux is produced by blazars.

In 2017, TXS 0506+056 was the first flaring blazar to be associated with the high-energy neutrino IC-190730A [4]. It was also the first astrophysical source to be associated with a highenergy neutrino at the $3 \sigma$ level. Evidence for additional neutrinos was found in archival IceCube data [61], the latter constitute $3.5 \sigma$ departure from background expectations. During the archival neutrino flare, TXS 0506+056 was not in a flaring $\gamma$-ray state.

Periods of flaring EM emission are promising as times of neutrino production. Qualitatively, during the flare, the target material (photons) is more abundant, and, likely, protons are also more abundant during such periods. Thus a non-linear increase of the neutrino flux is predicted in most theoretical models, e.g. [18, 19, 23]. Model predictions vary considerably for different blazars, and different models lead to expected neutrino counts in IceCube in the range $10^{-5}$ - few neutrinos per source e.g.[51,62] though models that predict more than a few neutrinos per source are already constrained by the absence of such signal in IceCube. An additional advantage of focusing on flaring emission is that the experimental search is more sensitive when the neutrino signal is transient. 

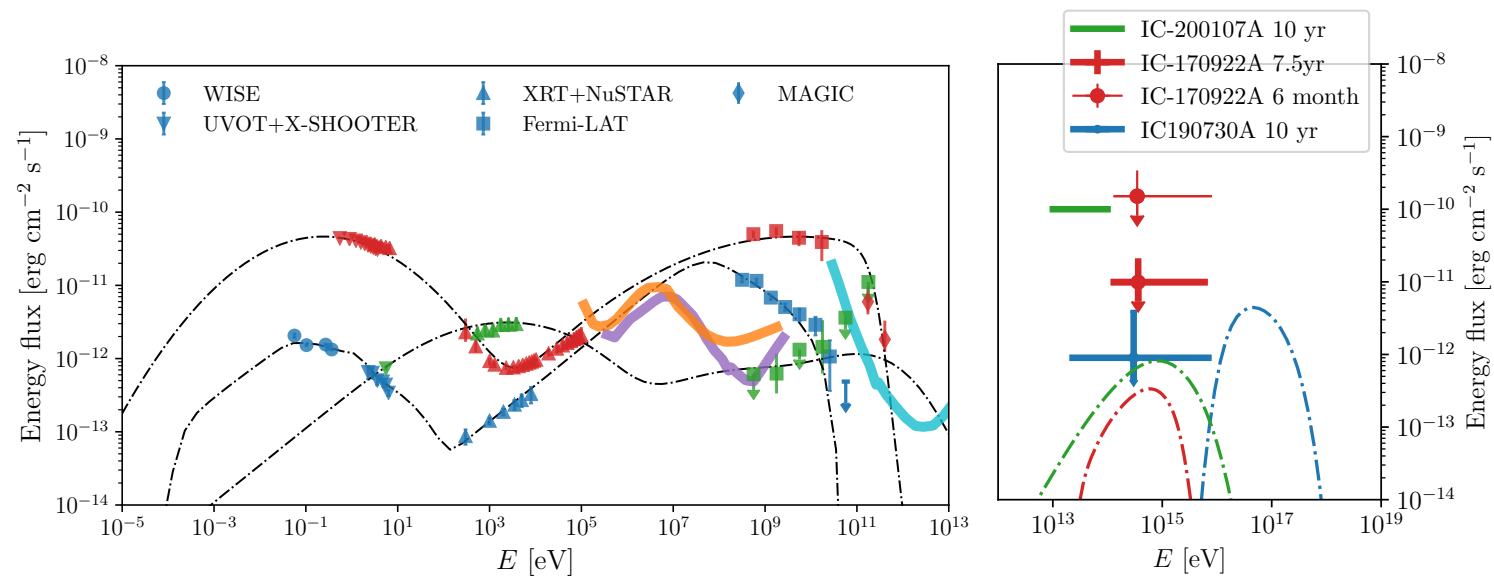

Figure 5: Left: Same as the Fig. 4 but shown in terms of the arriving energy flux. The orange line indicates the planned one-year sensitivity of eAstrogram in survey mode [54]. The purple line indicates the planned three-year sensitivity of AMEGO [55]. The blue curve indicates the planned sensitivity of CTA North after 50 hours of observation[56]. Right: Model-predicted neutrino energy flux for TXS 0506+056 (red dot-dashed), PKS 1502+106 (blue dot-dashed), and 3HSP J095507.9+355101 (green dot-dashed) at the time of arrival of of IC-190730A, IC-170922A, and IC-200107A respectively. The neutrino fluxes shown correspond to the most optimistic models of [51], [52] , and [53] respectively. Red, blue, and green arrows indicate the $90 \%$ CL upper limit on the neutrino flux derived from the detection of IC-190730A, IC-170922A and IC-200107A under the assumption of constant neutrino emission for six months (seven and a half years) - thin (thick) lines for IC-190730A, and ten years in the case of IC-200107A and IC-170922A.

Since the observation of IC-190730A, more blazars have been found in the error circles of high-energy alert neutrinos, albeit with lower significance individually. See e.g. [63] in these proceedings, as well as [64-68]. In [51-53], we have performed detailed leptohadronic modelling for three of these sources, in order to study the observed associations from the theoretical point of view. Fig. 4 shows the three studied blazars in terms of their intrinsic luminosities. PKS 1502+106 stands out in terms of its intrinsic $\gamma$-ray luminosity. 3HSP J095507.9+355101 stands out as it has a synchrotron peak at very high energy. Due to this observation, it is classified as an "extreme" blazar. In terms of their spectral energy distributions, the three sources are heterogeneous. Fig. 5 left, shows the spectral energy distributions in terms of the arriving energy flux at Earth. TXS 0506+056 had a much higher $\gamma$-ray flux at the time of the neutrino arrival than the other two sources. This explains the higher statistical significance of this association with respect to the other two.

\subsection{TXS 0506+056}

IC-190730A was detected with energy $~ 290 \mathrm{TeV}$. At the time of its arrival, TXS 0506+056, which lies inside the 50\% containment contour of the arrival direction of IC-190730A, was undergoing its most extreme recorded $\gamma$-ray flare. In [69], it was demonstrated that TXS 0506+056 is a misclassified BL Lac and is intrinsically of the flat spectrum radio quasar (FSRQ) type, meaning that it possesses an efficient accretion disk and that a dense ultraviolet photon field surrounds the black hole (the Broad Line Region). Such photon fields are optimal for the production of $\gtrsim \mathrm{PeV}$-energy neutrinos.

Several groups modelled this source, and independently concluded that the maximum neutrino 
emission during the six-month-long flare must have yielded $N_{v_{\mu}+\bar{v}_{\mu}} \leq 0.05$ in IceCube [31-34]. The upper limit is a consequence of the EM emission produced in $p \gamma$ interactions, which constitutes approximately 5/8ths of the total energy lost by protons in $p \gamma$ interactions as discussed in Section 2. Higher neutrino emission is not compatible with the observed EM emission of the source in the above-quoted models. The upper limit can be somewhat relaxed in the presence of multiple emission zones along the jet [70], or if matter obscures some of the EM emission [71].

The upper limit of $N_{v_{\mu}+\bar{v}_{\mu}} \leq 0.05$ means that we expect to observe one out of every 20 equally powerful neutrino flares on average [72]. However, the energetic requirements are extreme in order to achieve $N_{v_{\mu}+\bar{v}_{\mu}}=0.05$ in six months. The proton luminosity must be very high, with $\xi>1000$. Such values of $\xi$ and of $L_{p}$ are not representative of the blazar population [73].

For the archival neutrino flare of TXS 0506+056, most authors do not find a model that can produce sufficient neutrino flux to explain the observations [74, 75], see also [76]. But if certain conditions, which include a second, slower, jet layer surrounding the faster inner jet are present in TXS 0506+056, such neutrino flux could be achieved [77].

\subsection{HSP J095507.9+355101}

IC-200107A arrived from a direction where $90 \%$ of neutrinos have energy $0.33_{-0.27}^{+2.23} \mathrm{PeV}$ assuming an $E^{-2}$ neutrino spectrum. The extreme blazar 3HSP J095507.9+355101 ( $\left.\mathrm{z}=0.557\right)$ is inside the $90 \%$ containment region of the neutrino arrival direction. Target-of-opportunity observations revealed high, very hard, and variable X-ray emission from the source shortly after the neutrino arrival $[78,79]$. The X-ray high state lasted 44 days.

The leptohadronic modelling of [53] revealed that the Poisson probability to detect one neutrino from the source during the ten years of IceCube's operation is $\sim 3 \%$ for the most optimistic model studied, while detection of one neutrino during the 44-day-long high X-ray flux-state period following the neutrino detection is lower. The most promising scenarios for neutrino production also predict strong intra-source $\gamma$-ray attenuation above $100 \mathrm{GeV}$. If the association is real, then IceCube-Gen 2 and other future detectors should be able to provide additional evidence for neutrino production in other extreme blazars with similar $\gamma$-ray behaviour.

\subsection{PKS 1502+106}

PKS $1502+106$ is a very luminous source at large redshift $(z=1.84)$. During 2008-9 it underwent a strong $\gamma$-ray outburst. During that time, PKS $1502+106$ was the second brightest extragalactic $\gamma$-ray source. IC-170922A has most probable energy $\sim 300 \mathrm{TeV}$. At the time of arrival of IC-170922A, the source was quiet in the $\gamma$-ray energy range.

Models of PKS 1502+106 can easily account for the detection of several neutrinos during the lifetime of IceCube, if the emitting region of the neutrinos and $\gamma$-rays is close to the SMBH which is surrounded by dense radiation fields [80], related to the powerful accretion disk that it possesses. However, radio interferometric and $\gamma$-ray observations suggest that the emitting region is more likely at a larger distance to the base of the jet [81]. Taking these observations into account, we showed in [52], that PKS 1502+106 can have produced up to of order $N_{v_{\mu}+\bar{v}_{\mu}} \sim 0.1$ neutrinos during the ten-year operation of IceCube. An appealing feature of this model is that the required proton luminosity is consistent with the upper limits on the baryon loading factor of the entire blazar population. 


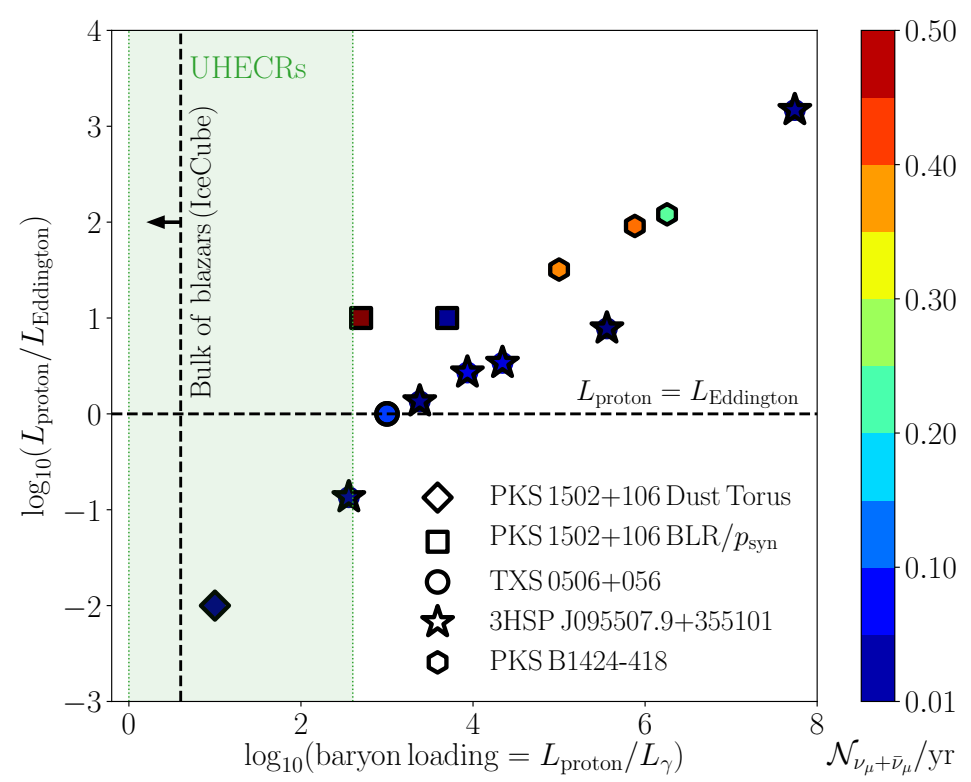

Figure 6: Baryon loading factor, defined as the ratio of the proton luminosity to the total electromagnetic radiation of the jet, versus ratio of proton to Eddington luminosity in the most optimistic leptohadronic models of PKS 1502+106, PKS B1424-418, 3HSP J095507.9+355101, and TXS 0506+056. The model predictions listed are from [52] (PKS 1502+106 longterm emission, dust torus model-blue diamond), [80] (PKS 1502+106 longterm emission, broad line region model-red square, and proton synchrotron model-blue square), [34] (TXS 0506+056 longterm emission and emission during 2017 flare - blue circle), [23] (PKS B1424-418 longterm emission and emission during 2014 flare-hexagons), and [53] (3HSP J095507.9+355101 X-ray flare and longterm emission-stars). Models above the horizontal dashed line assume proton luminosity which exceeds the Eddington luminosity of the SMBH. The vertical dashed line denotes the upper limit on the value of the baryon loading factor obtained by the EHE IceCube analysis for the entire blazar population [82] (recently updated in [3]) based on the model of [18]. The green band indicates the values of the baryon loading factor with which jetted AGN reproduce the observed UHECR spectrum [26].

\subsection{Implications}

Fig. 6 summarises the required proton content of the blazar jets in the three aforementioned blazars while maximising the expected number of neutrinos which is in the range $0.01-0.5 /$ year. Additionally, the modelling results of [23] on PKS B1424-418, which was found to be coincident with the cascade event IC-35 are shown [83]. The abscissa shows the required baryon-loading factor for a given neutrino flux to have been produced while the ordinate shows the required proton luminosity for each of the sources in terms of the Eddington luminosity of the SMBH. The majority of the models require super-Eddington proton luminosity and baryon loading which is larger than what is inferred from the level of the diffuse neutrino flux measured with IceCube. The baryonloading factor of extragalactic high-energy sources is independently constrained from UHECR observations. Fits to the UHECR data assuming that they all originate in blazar jets allow $\xi<400$, which is lower than what is required to account for the neutrino flux implied by the aforementioned blazar-alert-neutrino associations $[18,26]$. On the other hand, the neutrino models summarised in Fig. 6 above are not directly constrained by UHECR observations, as they don't assume cosmic-ray acceleration to ultra-high energies. 


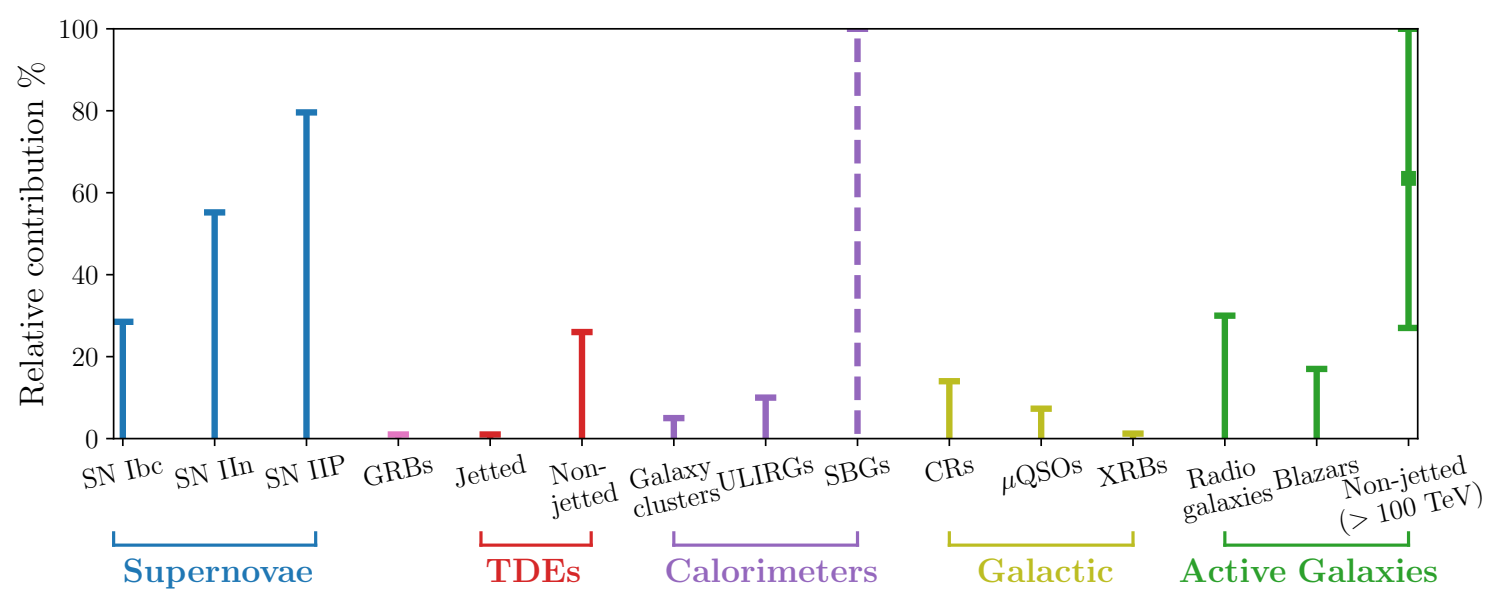

Figure 7: Bar chart of the possible contribution of different astrophysical source classes to the astrophysical neutrino flux measured by IceCube based on stacking analyses. The quoted values are $90 \%$ CL upper limits, except in the case of non-jetted AGN where the stacking analysis has led to a $2.6 \sigma$ signal and where the flux-fraction implied if the signal is genuine and associated $1 \sigma$ uncertainty are shown. See main text for details and for some of the caveats of these results.

Such proton luminosity implies, additionally, that the power of the relativistic jet is dominated by the power of relativistic protons and that the total jet power is one to two orders of magnitude higher than was previously inferred for the bulk of blazars [84] as discussed in [75, 85]. Such enormous jet power can only be accommodated if the accretion efficiency in the presence of a disk with a particular radiative luminosity was previously overestimated in standard accretion models. All in all, if the studied associations that point to super-Eddington proton luminosities are real, they are pointing to extreme events in the lifetime of the blazars. However, the required proton power seems unnaturally high and is in tension with the population-wide limits inferred from IceCube observations.

\section{Discussion and Outlook}

With blazars constrained as the main sources of the high-energy neutrino flux, the quest for the dominant neutrino source class continues. Fig. 7 summarises the possible contribution of different astrophysical source classes to the astrophysical neutrino flux based on the results of stacking analyses. Most of these analyses have resulted in upper limits. The upper limits are obtained based on different assumptions about the relative scaling of the neutrino flux with the EM fluxes of the source populations and are thus, necessarily, heterogeneous. The different analyses further suffer from heterogeneous choices of the minimum and maximum neutrino energy and of the assumed spectral index of the neutrino spectrum of the tested sources, which the conclusions of the analyses are very sensitive to. They should thus only be viewed as indicative. For source classes which are numerous with a positive redshift evolution, such as supernovae, catalogue completeness is likely very poor and thus the results of the stacking analyses of those objects should be considered as highly uncertain. 
The figure summarises $90 \%$ CL upper limits to the contribution of different source classes to the IceCube neutrino flux. The total contribution of different core-collapse supernovae ( $\mathrm{SNe}$ ) was constrained to be below $<55 \%$ (SNe IIn), $<80 \%$ (SNe IIP) and $<29 \%$ (SNe Ibc) of the total diffuse IceCube flux in [86], see also [87]. Core-collapse SNe could still produce $100 \%$ of the IceCube flux by summing the above upper limits, and the uncertainties related to catalogue completeness are severe for this source class, see also e.g. [88, 89]. Therefore, SNe are not constrained as the dominant sources of the IceCube neutrino flux. The prompt phase of $\gamma$-ray bursts (GRBs) has been constrained to account for $<1 \%$ of the high-energy neutrino flux [90]. Tidal disruption events (TDEs) produce no more than $26 \%$ of the high-energy neutrino intensity for non-jetted TDEs and $<1 \%$ for jettedTDEs [91]. Stacking upper limits have been obtained for various calorimetric environments, with ultra-luminous infrared galaxies (ULIRGs) constrained to $<10 \%$ [92], and galaxy clusters out to redshift, $z=2$ to $<5 \%$ [93]. The contribution of starburst galaxies (SBGs) has not been constrained by stacking analyses. Being a populous and strongly evolving source population, SBGs are difficult to constrain and by most arguments they are consistent with producing $100 \%$ of highenergy neutrinos (see however [89] for a different conclusion). Other stacking analyses constrain the contribution of Galactic sources to the high-energy neutrino flux. Galactic cosmic rays (CRs) can account for no more than $<14 \%$ [94], microquasars ( $\mu \mathrm{QSOs})<7 \%$, and TeV detected X-ray binaries $(\mathrm{XRBs})<1 \%$ [95]. Finally for AGN, stacking analyses constrain the relative contribution of blazars to $<17 \%$ [38], and radio galaxies to $<30 \%$ of the diffuse neutrino flux [96]. Recently, a $2.6 \sigma$ excess of neutrinos from the directions of non-jetted AGN has been reported [97]. If this is a genuine signal, it implies that $27 \%$ to $100 \%$ of neutrinos at $100 \mathrm{TeV}$ come from non-jetted AGN.

Blazars are the first well-studied extragalactic $\gamma$-ray emitting population and as such the most well-known class of extragalactic high-energy particle accelerators. All in all, the shape of the astrophysical neutrino spectrum, stacking limits, lack of point sources and absence of strong small-scale clustering point against blazars as the dominant source of high-energy astrophysical neutrinos. Theoretically, it is natural to expect that the blazar neutrino spectrum peaks beyond $10 \mathrm{PeV}$, as summarised in Section 2. Therefore, planned neutrino telescopes with peak sensitivity in the sub-EeV energy range such as Ashra-NTA, IceCube-Gen2 Radio, GRAND, POEMMA, RNO-G, Trinity, or Top-of-Mountain fluorescence telescope system will also probe, and possibly characterise the diffuse blazar neutrino flux [98-104].

Blazars remain testable neutrino point sources, especially during flares. Experimentally, shortduration flares are optimal for establishing neutrino emission from a point source. However, for the sources studied in detail thus far, it is not expected to detect sufficient neutrinos during a short-lived flare with a Gton neutrino detector, as the probability to detect neutrinos grows linearly with the neutrino fluence. Therefore, in the coming years, long-lived blazar flares will be most promising for establishing neutrino emission from blazars and thus the acceleration of high-energy hadrons in large-scale relativistic jets.

\section{Acknowledgements}

I would like to thank the ICRC organisers for the nice conference and for the invitation to give this talk. Thanks to Kohta Murase and Michael Unger for constructive comments on this manuscript. I also acknowledge my collaborators for the joint work that was summarised in this contribution. 


\section{References}

[1] ICECubE collaboration, First observation of PeV-energy neutrinos with IceCube, Phys.Rev.Lett. 111 (2013) 021103 [1304 . 5356].

[2] IcECuBe collaboration, Characteristics of the diffuse astrophysical electron and tau neutrino flux with six years of Ice Cube high energy cascade data, Phys. Rev. Lett. 125 (2020) 121104 [2001.09520].

[3] ICECube collaboration, Improved Characterization of the Astrophysical Muon-Neutrino Flux with 9.5 Years of IceCube Data, 2111.10299.

[4] IceCube, Fermi-LAT, MAGIC, AGILE, ASAS-SN, HAWC, H.E.S.S., INTEGRAL, Kanata, Kiso, Kapteyn, Liverpool Telescope, Subaru, Swift NuSTAR, VERITAS, VLA/17B-403 collaboration, Multimessenger observations of a flaring blazar coincident with high-energy neutrino IceCube-170922A, Science 361 (2018) eaat1378 [1807.08816].

[5] F. W. Stecker, Diffuse Fluxes of Cosmic High-Energy Neutrinos, Astrophys.J. 228 (1979) 919.

[6] V. Berezinsky, T. Gaisser, F. Halzen and T. Stanev, Diffuse radiation from cosmic ray interactions in the galaxy, Astropart.Phys. 1 (1993) 281.

[7] F. W. Stecker, C. Done, M. H. Salamon and P. Sommers, High-energy neutrinos from active galactic nuclei, Phys. Rev. Lett. 66 (1991) 2697.

[8] P. Mészáros and E. Waxman, $\mathrm{TeV}$ neutrinos from successful and choked gamma-ray bursts, Phys.Rev.Lett. 87 (2001) 171102 [astro-ph/0103275].

[9] A. Loeb and E. Waxman, The Cumulative background of high energy neutrinos from starburst galaxies, JCAP 0605 (2006) 003 [astro-ph/0601695].

[10] M. Ajello, R. Romani, D. Gasparrini, M. Shaw, J. Bolmer et al., The Cosmic Evolution of Fermi BL Lacertae Objects, Astrophys.J. 780 (2014) 73 [1310.0006].

[11] K. Mannheim, T. Stanev and P. L. Biermann, Neutrinos from flat-spectrum radio quasars, Astron. Astrophys. 260 (1992) L1.

[12] K. Mannheim, The proton blazar., Astron. Astrophys. 269 (1993) 67 [astro-ph/9302006].
[13] A. Atoyan and C. D. Dermer, High-energy neutrinos from photomeson processes in blazars, Phys.Rev.Lett. 87 (2001) 221102

[astro-ph/0108053].

[14] A. Muecke, R. J. Protheroe, R. Engel, J. P. Rachen and T. Stanev, BL Lac Objects in the synchrotron proton blazar model, Astropart. Phys. 18 (2003) 593 [astro-ph/0206164].

[15] A. M. Atoyan and C. D. Dermer, Neutral Beams from Blazar Jets, Astrophys. J. 586 (2003) 79 [astro-ph/0209231].

[16] A. Y. Neronov and D. V. Semikoz, Which blazars are neutrino loud?, Phys. Rev. D 66 (2002) 123003 [hep-ph/0208248].

[17] C. Dermer, E. Ramirez-Ruiz and T. Le, Correlation of Photon and Neutrino Fluxes in Blazars and Gamma Ray Bursts, Astrophys.J. 664 (2007) L67 [astro-ph/0703219].

[18] K. Murase, Y. Inoue and C. D. Dermer, Diffuse neutrino intensity from the inner jets of active galactic nuclei: Impacts of external photon fields and the blazar sequence, Phys. Rev. D 90 (2014) 023007 [1403 . 4089].

[19] F. Tavecchio, G. Ghisellini and D. Guetta, Structured Jets in BL Lac Objects: Efficient PeV Neutrino Factories?, Astrophys.J. 793 (2014) L18.

[20] M. Petropoulou, S. Dimitrakoudis, P. Padovani, A. Mastichiadis and E. Resconi, Photohadronic origin of $\gamma$-ray BL Lac emission: implications for IceCube neutrinos, MNRAS 448 (2015) 2412 [1501.07115].

[21] M. Petropoulou, S. Coenders and S. Dimitrakoudis, Time-dependent neutrino emission from Mrk 421 during flares and predictions for IceCube, Astropart. Phys. 80 (2016) 115 [1603.06954].

[22] P. Padovani, M. Petropoulou, P. Giommi and E. Resconi, A simplified view of blazars: the neutrino background, MNRAS 452 (2015) 1877 [1506.09135].

[23] S. Gao, M. Pohl and W. Winter, On the direct correlation between gamma-rays and PeV neutrinos from blazars, Astrophys. J. 843 (2017) 109 [1610.05306]. 
[24] X. Rodrigues, A. Fedynitch, S. Gao, D. Boncioli and W. Winter, Neutrinos and Ultra-High-Energy Cosmic-Ray Nuclei from Blazars, Astrophys. J. 854 (2018) 54 [1711.02091].

[25] A. Palladino, X. Rodrigues, S. Gao and W. Winter, Interpretation of the diffuse astrophysical neutrino flux in terms of the blazar sequence, Astrophys. $J$. 871 (2019) 41 [1806. 04769].

[26] X. Rodrigues, J. Heinze, A. Palladino, A. van Vliet and W. Winter, Active Galactic Nuclei Jets as the Origin of Ultrahigh-Energy Cosmic Rays and Perspectives for the Detection of Astrophysical Source Neutrinos at EeV Energies, Phys. Rev. Lett. 126 (2021) 191101 [2003.08392].

[27] K. Murase, F. Oikonomou and M. Petropoulou, Blazar Flares as an Origin of High-Energy Cosmic Neutrinos?, Astrophys. J. 865 (2018) 124 [1807.04748].

[28] E. Waxman and J. N. Bahcall, High-energy neutrinos from astrophysical sources: An Upper bound, Phys.Rev. D59 (1998) 023002 [hep-ph/9807282].

[29] K. Murase, D. Guetta and M. Ahlers, Hidden Cosmic-Ray Accelerators as an Origin of TeV-PeV Cosmic Neutrinos, Phys. Rev. Lett. 116 (2016) 071101 [1509.00805].

[30] M. Petropoulou and A. Mastichiadis, Bethe-Heitler emission in BL Lacs: filling the gap between $X$-rays and $\gamma$-rays, MNRAS 447 (2015) 36 [1411. 1908].

[31] MAGIC collaboration, The blazar TXS 0506+056 associated with a high-energy neutrino: insights into extragalactic jets and cosmic ray acceleration, Astrophys. J. Lett. 863 (2018) L10 [1807 . 04300].

[32] M. Cerruti, A. Zech, C. Boisson, G. Emery, $\mathrm{S}$. Inoue and J. P. Lenain, Lepto-hadronic single-zone models for the electromagnetic and neutrino emission of TXS 0506+056, MNRAS 483 (2019) L12 [1807. 04335].

[33] S. Gao, A. Fedynitch, W. Winter and M. Pohl, Modelling the coincident observation of a high-energy neutrino and a bright blazar flare, Nat. Astron. 3 (2019) 88 [1807. 04275].

[34] A. Keivani et al., A Multimessenger Picture of the Flaring Blazar TXS 0506+056: implications for High-Energy Neutrino Emission and Cosmic Ray
Acceleration, Astrophys. J. 864 (2018) 84

[1807.04537].

[35] IceCube collaboration, Measurement of the Diffuse Astrophysical Muon-Neutrino Spectrum with Ten Years of IceCube Data, PoS ICRC2019 (2020) 1017 [1908.09551].

[36] ICECube collaboration, Differential limit on the extremely-high-energy cosmic neutrino flux in the presence of astrophysical background from nine years of IceCube data, Phys. Rev. D98 (2018) 062003 [1807.01820].

[37] E. Zas, Searches for neutrino fluxes in the EeV regime with the Pierre Auger Observatory, PoS ICRC2017 (2017) 972.

[38] IceCube collaboration, Searches for steady neutrino emission from 3 FHLblazars using eight years of IceCube data from theNorthern hemisphere, PoS ICRC2019 (2020) 916 [1908.08458].

[39] IceCube collaboration, The IceCube high-energy starting event sample: Description and flux characterization with 7.5 years of data, Phys. Rev. D 104 (2021) 022002 [2011.03545].

[40] Fermi-LAT collaboration, 3FHL: The Third Catalog of Hard Fermi-LAT Sources, Astrophys. J., Suppl. Ser. 232 (2017) 18 [1702 . 00664].

[41] K. Murase and E. Waxman, Constraining High-Energy Cosmic Neutrino Sources: Implications and Prospects, Phys. Rev. D94 (2016) 103006 [1607 . 01601].

[42] S. Ando, M. R. Feyereisen and M. Fornasa, How bright can the brightest neutrino source be?, Phys. Rev. D95 (2017) 103003 [1701.02165].

[43] C. Yuan, K. Murase and P. Mészáros, Complementarity of Stacking and Multiplet Constraints on the Blazar Contribution to the Cumulative High-energy Neutrino Intensity, Astrophys. J. 890 (2020) 25 [1904.06371].

[44] F. Capel, D. J. Mortlock and C. Finley, Bayesian constraints on the astrophysical neutrino source population from IceCube data, Phys. Rev. D 101 (2020) 123017 [2005.02395].

[45] E. Waxman, IceCube's Neutrinos: The beginning of extra-Galactic neutrino astrophysics?, 1312.0558. 
[46] M. Ahlers and F. Halzen, Opening a New Window onto the Universe with IceCube, Prog. Part. Nucl. Phys. 102 (2018) 73 [1805.11112].

[47] K. Murase and M. Fukugita, Energetics of High-Energy Cosmic Radiations, Phys. Rev. D 99 (2019) 063012 [1806. 04194].

[48] Fermi LAT Collaboration collaboration, Resolving the Extragalactic $\gamma$-Ray Background above $50 \mathrm{GeV}$ with the Fermi Large Area Telescope, Phys. Rev. Lett. 116 (2016) 151105 [1511.00693].

[49] M. Lisanti, S. Mishra-Sharma, L. Necib and B. R. Safdi, Deciphering Contributions to the Extragalactic Gamma-Ray Background from $2 \mathrm{GeV}$ to $2 \mathrm{TeV}$, Astrophys. J. 832 (2016) 117 [1606.04101].

[50] H.-S. Zechlin, A. Cuoco, F. Donato, N. Fornengo and M. Regis, Statistical Measurement of the Gamma-ray Source-count Distribution as a Function of Energy, Astrophys. J. Lett. 826 (2016) L31 [1605. 04256].

[51] F. Oikonomou, K. Murase, P. Padovani, E. Rescon and P. Mészáros, High energy neutrino flux from individual blazar flares, MNRAS 489 (2019) 4347 [1906.05302].

[52] F. Oikonomou, M. Petropoulou, K. Murase, A. Tohuvavohu, G. Vasilopoulos, S. Buson et al., Multi-messenger emission from the parsec-scale jet of the flat-spectrum radio quasar coincident with high-energy neutrino IceCube-190730A, JCAP 10 (2021) 082 [2107. 11437].

[53] M. Petropoulou, F. Oikonomou, A. Mastichiadis, K. Murase, P. Padovani, G. Vasilopoulos et al., Comprehensive Multimessenger Modeling of the Extreme Blazar 3HSP J095507.9+355101 and Predictions for IceCube, Astrophys. J. 899 (2020) 113 [2005.07218].

[54] E-ASTROGAM collaboration, The e-ASTROGAM mission, Exper. Astron. 44 (2017) 25 [1611.02232].

[55] AMEGO collaboration, All-sky Medium Energy Gamma-ray Observatory: Exploring the Extreme Multimessenger Universe, 1907.07558.

[56] “CTAO's expected performance." https://www. cta-observatory.org/science/ctao-performance/.
[57] A. Plavin, Y. Y. Kovalev, Y. A. Kovalev and S. Troitsky, Observational Evidence for the Origin of High-energy Neutrinos in Parsec-scale Nuclei of Radio-bright Active Galaxies, Astrophys. J. 894 (2020) 101 [2001.00930].

[58] P. Giommi, T. Glauch, P. Padovani, E. Resconi, A. Turcati and Y. L. Chang, Dissecting the regions around IceCube high-energy neutrinos: growing evidence for the blazar connection, Mon. Not. Roy. Astron. Soc. 497 (2020) 865 [2001.09355].

[59] T. Hovatta, E. Lindfors, S. Kiehlmann, W. Max-Moerbeck, M. Hodges, I. Liodakis et al., Association of IceCube neutrinos with radio sources observed at Owens Valley and Metsähovi Radio Observatories, Astron. Astrophys. 650 (2021) A83 [2009. 10523].

[60] A. Franckowiak et al., Patterns in the Multiwavelength Behavior of Candidate Neutrino Blazars, Astrophys. J. 893 (2020) 162 [2001.10232].

[61] ICECuBE collaboration, Neutrino emission from the direction of the blazar TXS 0506+056 prior to the IceCube-170922A alert, Science 361 (2018) 147 [1807.08794].

[62] S. I. Stathopoulos, M. Petropoulou, P. Giommi, G. Vasilopoulos, P. Padovani and A. Mastichiadis, High-energy neutrinos from X-rays flares of blazars frequently observed by the Swift X-Ray Telescope, MNRAS (2021) [2111.09320].

[63] Fermi-LAT collaboration, Fermi-LAT realtime follow-ups of high-energy neutrino alerts, PoS ICRC2021 (2021) 956 [2112.11586].

[64] V. A. Acciari, S. Ansoldi, L. A. Antonelli, A. Arbet Engels, M. Artero, K. Asano et al., Searching for VHE gamma-ray emission associated with IceCube neutrino alerts using FACT, H.E.S.S., MAGIC, and VERITAS, POS ICRC2021 (2021) 960.

[65] R. Abbasi, M. Ackermann, J. Adams, J. Aguilar, M. Ahlers, M. Ahrens et al., A model-independent analysis of neutrino flares detected in IceCube from $X$-ray selected blazars, PoS ICRC2021 (2021) 971.

[66] J. Aublin and A. Plavin, Search for an association between neutrinos and radio-selected blazars with ANTARES, PoS ICRC2021 (2021) 1164. 
[67] G. Illuminati and A. Plavin, ANTARES search for neutrino flares from the direction of radio-bright blazars, PoS ICRC2021 (2021) 972.

[68] M. Kadler, U. Bach, D. Berge, S. Buson, D. Dorner, P. G. Edwards et al., TELAMON: Monitoring of AGN with the Effelsberg 100-m Telescope in the Context of Astroparticle Physics, PoS ICRC2021 (2021) 974.

[69] P. Padovani, F. Oikonomou, M. Petropoulou, P. Giommi and E. Resconi, TXS 0506+056, the first cosmic neutrino source, is not a BL Lac, MNRAS 484 (2019) L104 [1901. 06998].

[70] R. Xue, R.-Y. Liu, M. Petropoulou, F. Oikonomou, Z.-R. Wang, K. Wang et al., A two-zone model for blazar emission: implications for TXS 0506+056 and the neutrino event IceCube-170922A, Astrophys. J. 886 (2019) 23 [1908 . 10190].

[71] R.-Y. Liu, K. Wang, R. Xue, A. M. Taylor, X.-Y. Wang, Z. Li et al., Hadronuclear interpretation of a high-energy neutrino event coincident with a blazar flare, Phys. Rev. D99 (2019) 063008 [1807.05113].

[72] N. L. Strotjohann, M. Kowalski and A. Franckowiak, Eddington bias for cosmic neutrino sources, Astron. Astrophys. 622 (2019) L9 [1809.06865].

[73] IceCube collaboration, Constraints on Ultrahigh-Energy Cosmic-Ray Sources from a Search for Neutrinos above $10 \mathrm{PeV}$ with IceCube, Phys. Rev. Lett. 117 (2016) 241101 [1607.05886].

[74] X. Rodrigues, S. Gao, A. Fedynitch, A. Palladino and W. Winter, Leptohadronic blazar models applied to the 2014-15 flare of TXS 0506+056, Astrophys. J. 874 (2019) L29 [1812. 05939].

[75] M. Petropoulou et al., Multi-Epoch Modeling of TXS 0506+056 and Implications for Long-Term High-Energy Neutrino Emission, Astrophys. J. 891 (2020) 115 [1911.04010].

[76] A. Reimer, M. Boettcher and S. Buson, Cascading Constraints from Neutrino-emitting Blazars: The Case of TXS 0506+056, Astrophys. J. 881 (2019) 46 [1812.05654].

[77] B. T. Zhang, M. Petropoulou, K. Murase and F. Oikonomou, A Neutral Beam Model for High-Energy Neutrino Emission from the Blazar
TXS 0506+056, Astrophys. J. 889 (2020) 118 [1910.11464].

[78] P. Giommi, P. Padovani, F. Oikonomou, T. Glauch, S. Paiano and E. Resconi, $3 H S P$ J095507.9+355101: a flaring extreme blazar coincident in space and time with IceCube-200107A, Astron. Astrophys. 640 (2020) L4 [2003. 06405].

[79] V. S. Paliya, M. Boettcher, M. Gurwell and C. S. Stalin, On the Origin of Gamma-Ray Flares from Bright Fermi Blazars, Astrophys. J. Supp. 257 (2021) 37 [2111.04379].

[80] X. Rodrigues, S. Garrappa, S. Gao, V. S. Paliya, A. Franckowiak and W. Winter, Multiwavelength and Neutrino Emission from Blazar PKS $1502+$ 106, Astrophys. J. 912 (2021) 54 [2009. 04026].

[81] V. Karamanavis, L. Fuhrmann, E. Angelakis, I. Nestoras, I. Myserlis, T. P. Krichbaum et al., What can the 2008/10 broadband flare of PKS $1502+106$ tell us?. Nuclear opacity, magnetic fields, and the location of $\gamma$ rays, Astron. Astrophys. 590 (2016) A48 [1603. 04220].

[82] M. G. Aartsen, K. Abraham, M. Ackermann, J. Adams, J. A. Aguilar, M. Ahlers et al., Constraints on Ultrahigh-Energy Cosmic-Ray Sources from a Search for Neutrinos above $10 \mathrm{PeV}$ with IceCube, Phys. Rev. Lett. 117 (2016) 241101 [1607.05886]

[83] M. Kadler et al., Coincidence of a high-fluence blazar outburst with a PeV-energy neutrino event, Nature Phys. 12 (2016) 807 [1602 . 02012].

[84] G. Ghisellini, F. Tavecchio, L. Maraschi, A. Celotti and T. Sbarrato, The power of relativistic jets is larger than the luminosity of their accretion disks, Nature 515 (2014) 376 [1411.5368].

[85] G. Ghisellini, Extra-galactic jets: a hard X-ray view, Mem. Soc. Ast. It. 90 (2019) 154 [1911.11777].

[86] ICECube collaboration, Searching for High-Energy Neutrinos from Core-Collapse Supernovae with IceCube, PoS ICRC2021 (2021) 1116 [2107.09317].

[87] A. J. Stasik, Search for high energetic neutrinos from core collapse supernovae using the IceCube neutrino telescope, Ph.D. thesis, Humboldt University of Berlin, Germany, Jan., 2018. 
[88] ICECube collaboration, Constraints on minute-scale transient astrophysical neutrino sources, Phys. Rev. Lett. 122 (2019) 051102 [1807.11492].

[89] I. Bartos, D. Veske, M. Kowalski, Z. Marka and S. Marka, The IceCube Pie Chart: Relative Source Contributions to the Cosmic Neutrino Flux, Astrophys. J. 921 (2021) 45 [2105.03792].

[90] IcECube collaboration, An All-Sky Search for Three Flavors of Neutrinos from Gamma-Ray Bursts with the IceCube Neutrino Observatory, Astrophys. J. 824 (2016) 115 [1601.06484].

[91] ICECube collaboration, Search for Neutrinos from Populations of Optical Transients, PoS ICRC2019 (2020) 1016 [1908 .08547].

[92] ICECUBE collaboration, Search for High-Energy Neutrinos from Ultra-Luminous Infrared Galaxies with Ice Cube, arXiv e-prints (2021) [2107.03149].

[93] ICECuBE collaboration, A time-independent search for neutrinos from galaxy clusters with IceCube, PoS ICRC2021 (2021) 1133 [2107. 10080].

[94] M. G. Aartsen, M. Ackermann, J. Adams, J. A. Aguilar, M. Ahlers, M. Ahrens et al., Constraints on Galactic Neutrino Emission with Seven Years of IceCube Data, Astrophys. J. 849 (2017) 67 [1707.03416].

[95] ICECube collaboration, Searching for time-dependent high-energy neutrino emission from X-ray binaries with IceCube, PoS ICRC2021 (2021) 1136 [2107.12383].

[96] B. Zhou, M. Kamionkowski and Y.-f. Liang, Search for High-Energy Neutrino Emission from
Radio-Bright AGN, Phys. Rev. D 103 (2021) 123018 [2103. 12813].

[97] ICECube collaboration, A search for neutrino emission from cores of Active Galactic Nuclei, 2111. 10169.

[98] NTA collaboration, Neutrino Telescope Array (NTA): Multi-Astroparticle Explorer for PeV-EeV Universe-For Clear Identification of Cosmic Accelerators and Cosmic Beam Physics-, PoS ICRC2017 (2018) 941.

[99] IceCube-Gen2 collaboration, IceCube-Gen2: the window to the extreme Universe, J. Phys. G 48 (2021) 060501 [2008.04323].

[100] GRAND collaboration, The Giant Radio Array for Neutrino Detection (GRAND): Science and Design, Sci. China Phys. Mech. Astron. 63 (2020) 219501 [1810.09994].

[101] POEMMA collaboration, The POEMMA (Probe of Extreme Multi-Messenger Astrophysics) observatory, JCAP 06 (2021) 007 [2012.07945].

[102] RNO-G collaboration, Design and Sensitivity of the Radio Neutrino Observatory in Greenland (RNO-G), JINST 16 (2021) P03025 [2010 . 12279].

[103] A. M. Brown, M. Bagheri, M. Doro, E. Gazda, D. Kieda, C. Lin et al., Trinity: an imaging air Cherenkov telescope to search for Ultra-High-Energy neutrinos, PoS ICRC2021 (2021) 1179.

[104] A. Neronov, Sensitivity of top-of-the-mountain fluorescence telescope system for astrophysical neutrino flux above $10 \mathrm{PeV}$, Astropart. Phys. 128 (2021) 102549 [1905 . 10606]. 